\title{
Syntrophic butyrate and propionate oxidation processes: from genomes to reaction mechanisms
}

\author{
Nicolai Müller, ${ }^{1 \dagger}$ Petra Worm, ${ }^{2 \dagger}$ Bernhard Schink, ${ }^{1 \star}$ \\ Alfons J. M. Stams ${ }^{2}$ and Caroline M. Plugge ${ }^{2}$ \\ ${ }^{1}$ Faculty for Biology, University of Konstanz, D-78457 \\ Konstanz, Germany. \\ ${ }^{2}$ Laboratory of Microbiology, Wageningen University, \\ Dreijenplein 10, 6703 HB Wageningen, the Netherlands.
}

\section{Summary}

In anoxic environments such as swamps, rice fields and sludge digestors, syntrophic microbial communities are important for decomposition of organic matter to $\mathrm{CO}_{2}$ and $\mathrm{CH}_{4}$. The most difficult step is the fermentative degradation of short-chain fatty acids such as propionate and butyrate. Conversion of these metabolites to acetate, $\mathrm{CO}_{2}$, formate and hydrogen is endergonic under standard conditions and occurs only if methanogens keep the concentrations of these intermediate products low. Butyrate and propionate degradation pathways include oxidation steps of comparably high redox potential, i.e. oxidation of butyryl-CoA to crotonyl-CoA and of succinate to fumarate, respectively, that require investment of energy to release the electrons as hydrogen or formate. Although investigated for several decades, the biochemistry of these reactions is still not completely understood. Genome analysis of the butyrateoxidizing Syntrophomonas wolfei and Syntrophus aciditrophicus and of the propionate-oxidizing Syntrophobacter fumaroxidans and Pelotomaculum thermopropionicum reveals the presence of energytransforming protein complexes. Recent studies indicated that $S$. wolfei uses electron-transferring flavoproteins coupled to a menaquinone loop to drive butyryl-CoA oxidation, and that $S$. fumaroxidans uses a periplasmic formate dehydrogenase, cytochrome $b$ :quinone oxidoreductases, a menaquinone loop and

Received 3 November, 2009; accepted 9 January, 2010. *For correspondence. E-mail Bernhard.Schink@uni-konstanz.de; Tel. (+49) 7531 882140; Fax (+49) 7531884047 . 'Both authors contributed equally.

C 2010 Society for Applied Microbiology and Blackwell Publishing Ltd a cytoplasmic fumarate reductase to drive energydependent succinate oxidation. Furthermore, we propose that homologues of the Thermotoga maritima bifurcating [ $\mathrm{FeFe}$-hydrogenase are involved in NADH oxidation by $S$. wolfei and $S$. fumaroxidans to form hydrogen.

\section{Introduction}

In anoxic environments such as swamps, rice paddy fields and intestines of higher animals, methanogenic communities are important for decomposition of organic matter to $\mathrm{CO}_{2}$ and $\mathrm{CH}_{4}$ (Schink and Stams, 2006; Mcinerney et al., 2008; Stams and Plugge, 2009). Moreover, they are the key biocatalysts in anaerobic bioreactors that are used worldwide to treat industrial wastewaters and solid wastes. Different types of anaerobes have specified metabolic functions in the degradation pathway and depend on metabolite transfer which is called syntrophy (Schink and Stams, 2006). The study of syntrophic cooperation is essential to understand methanogenic conversions in different environments (Mcinerney et al., 2008). The most difficult step in this degradation is the conversion of short-chain fatty acids such as propionate and butyrate. Under standard conditions $\left(\mathrm{P}_{\mathrm{H} 2}\right.$ of $1 \mathrm{~atm}$, substrate and product concentrations of $1 \mathrm{M}$, temperature $298^{\circ} \mathrm{K}$ ), propionate and butyrate oxidation to $\mathrm{H}_{2}$, formate and acetate are endergonic reactions (Table 1). In anoxic environments, methanogenic Archaea maintain low $\mathrm{H}_{2}$, formate and acetate concentrations which make propionate and butyrate degradation feasible (Stams and Plugge, 2009). Syntrophic propionate and butyrate oxidations involve energy-dependent reactions that are biochemically not fully understood. However, recently several novel reactions were discussed to perform energy transformation in other bacteria. These reactions will be summarized in this report with respect to their possible implications in syntrophic fatty acid oxidation. Moreover, the genomes of two propionate degraders (Syntrophobacter fumaroxidans and Pelotomaculum thermopropionicum) and two butyrate degraders (Syntrophomonas wolfei and Syntrophus aciditrophicus) have been sequenced (Mcinerney et al., 2007; Kosaka et al., 2008). 
Table 1. Standard free reaction enthalpies of fatty acid oxidation and methane production.

\begin{tabular}{llc}
\hline Reaction & & $\Delta G^{0 \prime}$ (kJ per reaction) \\
\hline Propionate $^{-}+2 \mathrm{H}_{2} \mathrm{O} \rightarrow$ Acetate $^{-}+\mathrm{CO}_{2}+3 \mathrm{H}_{2}$ & Eq. 1 & +76.0 \\
Propionate $+2 \mathrm{H}_{2} \mathrm{O}+2 \mathrm{CO}_{2} \rightarrow$ Acetate $^{-}+3 \mathrm{HCOO}^{-}+3 \mathrm{H}^{+}$ & Eq. 1a & +65.3 \\
Butyrate $^{-}+2 \mathrm{H}_{2} \mathrm{O} \rightarrow 2 \mathrm{Acetate}^{-}+\mathrm{H}^{+}+2 \mathrm{H}_{2}$ & Eq. 2 & +48.3 \\
Butyrate $^{-} 2 \mathrm{H}_{2} \mathrm{O}+2 \mathrm{CO}_{2} \rightarrow 2 \mathrm{Acetate}^{-}+2 \mathrm{HCOO}^{-}+2 \mathrm{H}^{+}$ & Eq. 2a & +38.5 \\
$4 \mathrm{H}_{2}+\mathrm{CO}_{2} \rightarrow \mathrm{CH}_{4}+2 \mathrm{H}_{2} \mathrm{O}$ & Eq. 3 & -131.7 \\
$4 \mathrm{HCOO}^{-}+4 \mathrm{H}^{+} \rightarrow \mathrm{CH}_{4}+3 \mathrm{CO}_{2}+2 \mathrm{H}_{2} \mathrm{O}$ & Eq. 4 & -144.5 \\
$\mathrm{CH}_{3} \mathrm{COO}^{-}+\mathrm{H}^{+} \rightarrow \mathrm{CH}_{4}+\mathrm{CO}_{2}$ & Eq. 5 & -36 \\
\hline
\end{tabular}

Values calculated from the standard free formation enthalpies of the reactants at a concentration of $1 \mathrm{M}, \mathrm{pH} 7.0$ and $\mathrm{T}=25^{\circ} \mathrm{C}$ according to Thauer and colleagues (1977)

Based on genome analysis we propose that novel energytransforming reactions are involved in syntrophic butyrate and propionate degradation.

\section{Topic 1. Known energy-conserving mechanisms in syntrophic butyrate and propionate degradation}

\section{Butyrate degradation}

Butyrate oxidizers known to date belong to two groups of bacteria within the family Syntrophomonadaceae and the order Syntrophobacterales. Formerly classified as Clostridia, the members of the family Syntrophomonadaceae have been reassigned to a new family within the order Clostridiales, based on their 16S rRNA sequence (Zhao et al., 1993). Members of this family are S. wolfei, Syntrophomonas bryantii [formerly Syntrophospora bryantii (Wu et al., 2006)], Syntrophomonas erecta, Syntrophomonas curvata, Syntrophomonas zehnderi and Thermosyntropha lipolytica.

The second group of syntrophic butyrate degraders belongs to the Syntrophobacterales, an order of the deltaproteobacteria subdivision. Organisms of this group are S. aciditrophicus and Syntrophus buswellii. Several other Syntrophus strains such as Syntrophus gentianae are able to oxidize benzoate or other aromatic compounds syntrophically but these processes are not considered in this article. Remarkably, all these organisms are restricted to the use of saturated or unsaturated fatty acids. Alternative substrates or alternative electron acceptors to grow these bacteria in pure culture have not been found yet for these two groups of butyrate-oxidizing bacteria.

In all known butyrate-oxidizing bacteria, the betaoxidation pathway is used (Wofford et al., 1986; Schink, 1997; Mcinerney et al., 2007). First, butyrate is activated to butyryl-CoA with acetyl-CoA by a CoA transferase. Further oxidation proceeds via crotonyl-CoA and 3-hydroxybutyryl-CoA to acetoacetyl-CoA which is cleaved to two acetyl-CoA moieties. One of these is invested in butyrate activation, and the other one forms ATP via phosphotransacetylase and acetate kinase
(Wofford et al., 1986). Electrons are released in the oxidation of butyryl-CoA to crotonyl-CoA and in the oxidation of 3-hydroxybutyryl-CoA to acetoacetyl-CoA at $-250 \mathrm{mV}$ (Gustafson et al., 1986). As the standard midpoint redox potentials of these reducing equivalents are too high for reduction of protons to form $\mathrm{H}_{2}[-414 \mathrm{mV}$ (Thauer et al., 1977; Schink, 1997)], it was postulated that an energydependent reversed electron transport is required to overcome the redox potential difference (Thauer and Morris, 1984). The partner organism keeps the hydrogen partial pressure low, thus raising the redox potential of proton reduction to a level around $-300 \mathrm{mV}$ (Schink, 1997). The butyrate-oxidizing organism has to sacrifice part of the gained ATP to shift electrons to this redox potential, and the remaining ATP can be used for biosynthesis and growth. As such fractional amounts of ATP cannot be provided by substrate level phosphorylation such energy transformations have to be coupled to the cytoplasmic membrane (Thauer and Morris, 1984). Indeed, hydrogen production from butyrate has been shown to be sensitive to the protonophore CCCP and the ATPase inhibitor DCCD, thus providing evidence for participation of a transmembrane proton potential (Wallrabenstein and Schink, 1994). However, the underlying biochemical mechanisms remained enigmatic until the completion of the genome sequence of $S$. aciditrophicus (Mcinerney et al., 2007). It was stated that electrons released in butyryl-CoA oxidation are transferred to components of the membrane where they reduce $\mathrm{NAD}^{+}$to $\mathrm{NADH}$ in an endergonic manner, e.g. through an rnf-coded oxidoreductase, and the necessary energy would be supplied by a sodium ion gradient which in turn is provided by ATP-dependent proton efflux and a sodium/proton antiporter. In S. wolfei, however, a different reaction mechanism has to be active since the genome of this bacterium does not contain rnf genes which will be discussed later in this review (Müller et al., 2009).

\section{Propionate degradation}

Several bacterial strains are known to degrade propionate in syntrophic association with methanogens: 
S. fumaroxidans, S. wolinii, S. pfennigii, S. sulfatireducens, P. thermopropionicum, P. schinkii, P. propionicicum, Smithella propionica and Desulfotomaculum thermobenzoicum ssp. thermosyntrophicum. These bacteria belong to the Syntrophobacterales, an order of the deltaproteobacteria subdivision, and to the family Peptococcaceae within the order Clostridiales. Smithella propionica converts propionate through a dismutating pathway to acetate and butyrate after which butyrate is oxidized to acetate (de Bok et al., 2001). All other known syntrophic propionate degraders oxidize propionate to acetate plus $\mathrm{CO}_{2}$. They use the methylmalonyl-CoA pathway which generates per molecule propionate one ATP via substrate level phosphorylation and three electron pairs by: (i) oxidation of succinate to fumarate $\left(E^{\circ \prime}=+30 \mathrm{mV}\right.$ ), (ii) oxidation of malate to oxaloacetate $\left(E^{\circ \prime}=-176 \mathrm{mV}\right)$, and (iii) pyruvate conversion to acetyl$\mathrm{CoA}$ and $\mathrm{CO}_{2}\left(\mathrm{E}^{\circ \prime}=-470 \mathrm{mV}\right)$ (Fig. 2). The latter step can easily be coupled to proton reduction $\left(\mathrm{E}^{\circ \prime}=\right.$ $-414 \mathrm{mV})$ or $\mathrm{CO}_{2} \quad\left(E^{\circ \prime}=-432 \mathrm{mV}\right)$ reduction (Thauer et al., 1977) via ferredoxin, as anaerobic bacteria generally contain pyruvate : ferredoxin oxidoreductases (Chabrière et al., 1999).

Oxidation of succinate and malate with protons would require hydrogen partial pressures of $10^{-15}$ and $10^{-8}$ atm respectively (Schink, 1997). Thauer and Morris (1984) and Schink (1997) proposed a reversed electron transport mechanism. The hydrolysis of 2/3 ATP coupled with a transmembrane import of two protons would make succinate oxidation energetically possible. Later, van Kuijk and colleagues (1998) proposed that this reaction is analogous to that involved in fumarate respiration by Wolinella succinogenes. This bacterium generates a transmembrane proton gradient by periplasmic hydrogen or formate oxidation coupled to cytoplasmic fumarate reduction via cytochromes and a menaquinone loop (Kröger et al., 2002). In S. fumaroxidans, fumarate reductase and succinate dehydrogenase activity are membrane bound. Hydrogenase and formate dehydrogenase activity are found in the periplasmic space loosely attached to the membrane, and cells contain cytochrome $c$ and $b$ and menaquinone-6 and -7 as possible electron carriers (van Kuijk et al., 1998). Syntrophobacter fumaroxidans appears to gain around 2/3 ATP per mol fumarate if $\mathrm{H}_{2}$ or formate is oxidized with fumarate. It was suggested that this mechanism in reverse could reduce periplasmic protons with the energy-dependent cytoplasmic succinate oxidation.

Malate oxidation to oxaloacetate $\left(E^{\circ \prime}=-176 \mathrm{mV}\right)$ is coupled to $\mathrm{NAD}^{+}$reduction $\left(\mathrm{E}^{\circ \prime}=-320 \mathrm{mV}\right)($ Van kuijk and Stams, 1996). Yet, the exact mechanism of NADH oxidation and terminal reduction of protons and/or $\mathrm{CO}_{2}$ in S. fumaroxidans remains unclear and deserves further investigation.

\section{Topic 2. Mechanisms for energy conservation in anaerobic microorganisms}

\section{Electron transport phosphorylation}

Electron transport phosphorylation is the most important energy-conserving mechanism in organisms that reduce external electron acceptors such as oxygen, nitrate, sulfate, etc. (Richardson, 2000). In most cases, the membrane-bound NADH dehydrogenase (complex I) oxidizes NADH with quinones in the membrane while translocating protons into the periplasmic space via a transmembrane proton pump (Richardson, 2000). The electrons are transferred further to the respective terminal acceptor via cytochromes.

Protons can be translocated to the periplasmic space by at least two possible mechanisms. The first one includes the translocation of protons or sodium ions through the transmembrane proton channel of an NADH dehydrogenase (Richardson, 2000). The second mechanism involves a redox loop and is supposed to be the most common way of proton translocation in bacteria (Richardson, 2000). Here, isoprenoid quinones within the membrane are reduced by the membrane-integral domain of the electron-donating enzyme, together with protons. The reduced quinone diffuses laterally through the membrane to the membrane domain of the accepting enzyme where electrons are transferred to an electron acceptor, and the protons are released at the opposite side of the membrane. An example is the redox loop of the formate dehydrogenase FDH-N coupled to nitrate reductase in Escherichia coli (Jormakka et al., 2002). During anaerobic growth, formate is oxidized in the periplasm by FDH-N while protons are transferred to menaquinones, together with the electrons released in formate oxidation. Subsequently, menaquinol is oxidized at the membrane domain of the nitrate reductase, releasing protons to the periplasm while electrons are transferred to nitrate to form nitrite at the cytoplasmic side of the membrane (Jormakka et al., 2002).

\section{The smallest quantum of energy in biology}

Since only small amounts of chemical energy can be transformed in syntrophic oxidation processes (Table 1), energy has to be efficiently conserved. Thauer and colleagues (1977) and Schink (1997) calculated that the minimal cost of synthesis of one ATP is $60 \mathrm{~kJ}$ per mol. Syntrophically fermenting bacteria such as butyrate and propionate oxidizers have to invest part of their ATP to create a proton gradient. For a long time it was thought that three protons are imported for synthesis of one ATP and thus the smallest quantum of energy that can be converted into ATP is in the range of $20 \mathrm{~kJ}$ per mol. However, Nakanishi-Matsui and Futai (2008) documented 
that the number of protons translocated is determined by the number of membrane-integral c-subunits of the ATP synthase which varies between different microorganisms. ATP synthases of yeast and Enterococcus hirae harbour 10, while Ilyobacter tartaricus, Methanopyrus kandleri and chloroplasts harbour 11,13 and 14 such subunits respectively. The authors proposed that with one full rotation of the ATP synthase complex, three ATP are hydrolysed, and each c-subunit translocates one proton. As a consequence, the number of protons translocated per ATP is between 3.3 and 4.6 and with this, the smallest quantum of biologically conservable energy may range from 13 to $18 \mathrm{~kJ}$ per mol reaction.

In reversed electron transport as hypothesized for syntrophic butyrate and propionate oxidation, a high number of protons transported per ATP hydrolysed would allow ATP synthesis even at low energy gains. Hoehler and colleagues (2001) calculated minimal amounts of -10 to $-19 \mathrm{~kJ}$ per translocated proton for organisms in anoxic methanogenic marine sediments. However, the number of membrane-integral c-subunits in ATP synthases of syntrophic bacteria has not been determined yet.

Genome analysis of $S$. fumaroxidans and $S$. wolfei indicates the presence of one kind of ATP synthase in each bacterium; the cytoplasmic F1 domain is encoded by Sfum_2581-2587 and Swol_2381-2385, and the membrane-integral $F_{0}$ domain is encoded by Sfum_16041605 and Swol_2387-2388 respectively. The ratio of transcription of $\mathrm{F} 1$ domain-coding genes to membraneintegral c-subunit-coding genes might give insight into the number of c-subunits per ATP synthase in $S$. fumaroxidans and $S$. wolfei in the future.

\section{Buckel-Thauer Bcd/Etf}

Most fermenting organisms have to regenerate their NAD pool in the absence of external electron acceptors. It was assumed that in clostridia $\mathrm{NADH}$ is oxidized with ferredoxin, which in turn is oxidized with protons to form hydrogen. This reaction is endergonic and, until recently, it was not known how hydrogen-producing microorganisms could perform such a reaction while strongly accumulating hydrogen in their environment.

Clostridium kluyveri ferments ethanol plus acetate to a mix of butyrate, caproate and hydrogen. The critical step of $\mathrm{NADH}$ oxidation with ferredoxin was recently found to be catalysed by a butyryl-CoA dehydrogenase (Bcd)/ electron-transferring flavoprotein subunit (Etf) complex which couples this endergonic reaction to the exergonic reduction of crotonyl-CoA to butyryl-CoA with NADH (Li et al., 2008). Overall, two NADH molecules are oxidized and one molecule reduced ferredoxin (transferring two electrons) plus one molecule butyryl-CoA are formed ( $\mathrm{Li}$ et al., 2008) which we refer to as bifurcation. For butyrate- oxidizing bacteria, a reversal of this reaction was suggested, i.e. the endergonic reduction of $\mathrm{NAD}^{+}$with butyrylCoA could be driven by the exergonic reduction of another $\mathrm{NAD}^{+}$with reduced ferredoxin (Herrmann et al., 2008), a reaction that we refer to as confurcation. This mechanism could provide a concept for the reversed electron transport in syntrophic fatty acid degradation since homologues of this enzyme complex were found in genomes of the syntrophs $S$. wolfei, $S$. fumaroxidans and $P$. thermopropionicum, but not in $S$. aciditrophicus.

\section{Confurcating/bifurcating [FeFe]-hydogenases}

Apart from the Buckel-Thauer Bcd/Etf complex, another enzyme with bifurcating/confurcating activity was described recently, the [FeFe]-hydrogenase of Thermotoga maritima (Schut and Adams, 2009). Thermotoga maritima ferments glucose to acetate, $\mathrm{CO}_{2}$ and $\mathrm{H}_{2}$ via the Embden-Meyerhof pathway which generates two NADH and four reduced ferredoxins per molecule of glucose. In order to re-oxidize these carriers, the proposed confurcating [FeFe]-hydrogenase uses simultaneously electrons from $\mathrm{NADH}$ and reduced ferredoxin in a $1: 2$ ratio to produce hydrogen (Schut and Adams, 2009). This hydrogenase could not use either NADH or reduced ferredoxin alone for hydrogen production. Additionally, the authors found genes with sequence similarity to this trimeric [FeFe]-hydrogenase also in other organisms such as S. fumaroxidans, $P$. thermopropionicum and S. wolfei (Table 2, Fig. S1). Remarkably, our gene analysis indicated that some putative [NiFe]-hydrogenases and formate dehydrogenases in $S$. fumaroxidans, $P$. thermopropionicum, $S$. wolfei and $S$. aciditrophicus contain subunits with iron-sulfur-binding motifs and subunits homologous with the $\mathrm{NADH}$ dehydrogenase $51 \mathrm{kDa}$ subunit, which is the NADH-binding subunit of Complex I. This indicates a possible confurcating function for [NiFe]hydrogenases and formate dehydrogenases as well.

\section{Rnf complex}

In Rhodobacter capsulatus nitrogen fixation ( $r n f$ genes were found which code for a membrane-bound enzyme complex that is most probably involved in energy transformation (Kumagai et al., 1997). Gene analysis indicated that the encoded products RnfB and RnfC contain ironsulfur clusters, RnfC contains potential NADH and FMN binding sites, and the membrane-bound RnfA, RnfD and RnfE are similar to subunits of the sodium-translocating NADH : quinone oxidoreductase (Kumagai et al., 1997). The authors proposed that this complex translocates protons or sodium ions to drive the endergonic reduction of ferredoxin by NADH oxidation. Analogous rnf genes were found in numerous bacteria such as Haemophilus influen- 


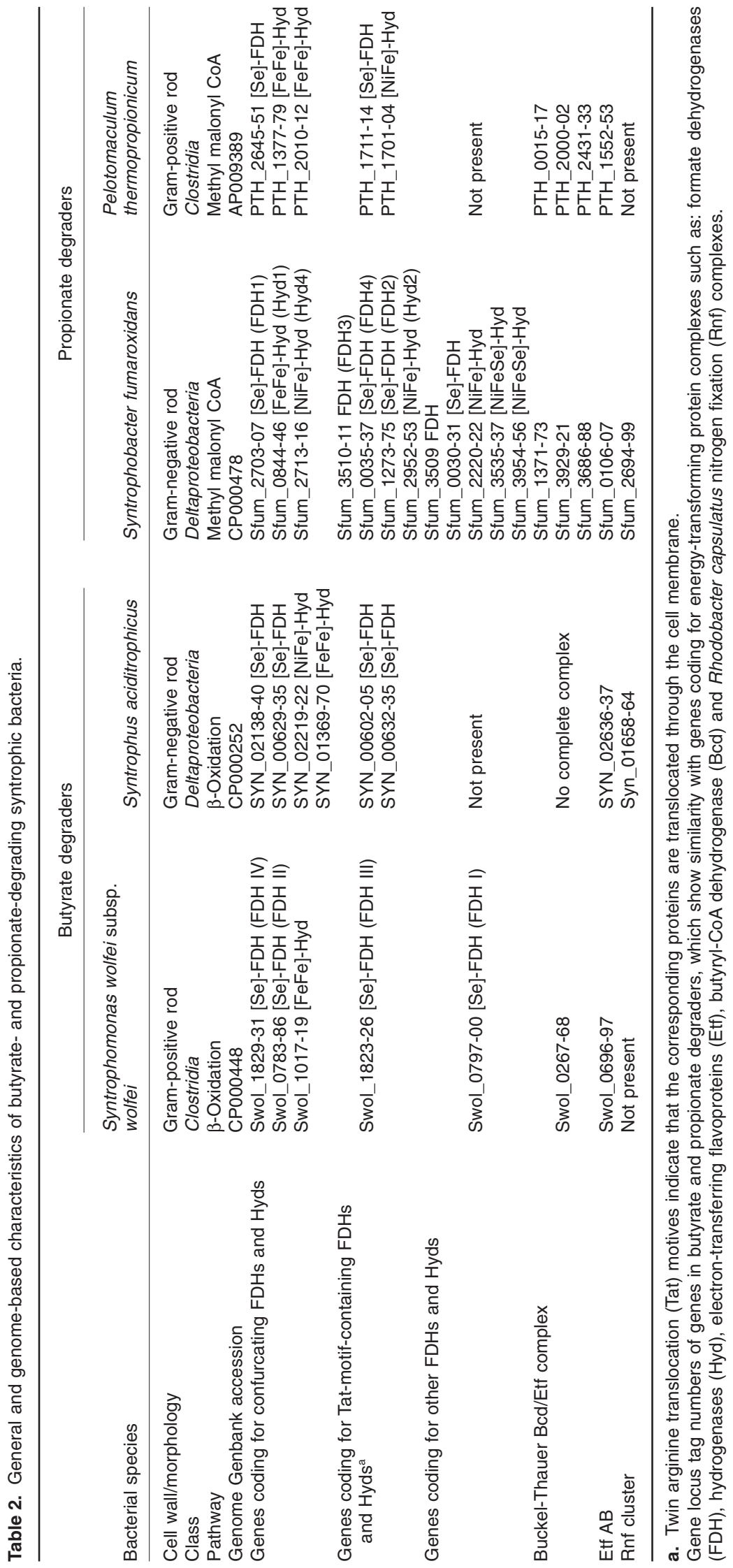


zae, E. coli, Acetobacterium woodii and Vibrio alginolyticus, thus suggesting a general and important function for the Rnf complex in energy conservation (Nakayama et al., 2000; Backiel et al., 2008; Müller et al., 2008).

Müller and colleagues (2008) investigated the function of an Rnf complex in the homoacetogenic bacterium $A$. woodii. Although biochemical proof has not been obtained yet, the authors found that caffeate respiration was coupled to ATP synthesis by a chemiosmotic mechanism with sodium ions as coupling ions, and that ferredoxin : $\mathrm{NAD}^{+}$-oxidoreductase was the only membrane-bound enzyme detected in the pathway of $\mathrm{H}_{2}$-dependent caffeate reduction. They postulated oxidation of ferredoxin with reduction of $\mathrm{NAD}^{+}$and the export of $\mathrm{Na}^{+}$.

\section{Methods used for gene analysis}

Automatic annotations of genomes from DOE-Joined Genome Institute (IMG-JGI-DOE, version 2.9 August 2009, http://www.jgi.doe.gov/) were used to indicate the presence of gene clusters coding for formate dehydrogenases, hydrogenases, Buckel-Thauer Bcd/Etf complexes and Rnf clusters in S. wolfei, S. aciditrophicus, P. thermopropionicum and $S$. fumaroxidans. $\mathrm{N}$-terminal amino acid sequences of FDH-1 and FDH-2 identified by de Bok and colleagues (2003) were used to find corresponding $f d h-1$ and $f d h-2$ nucleotide sequences in the genome of $S$. fumaroxidans. Pfam search (Sanger institute, 2009, http:// pfam.sanger.ac.uk/search) was used to identify motifs in the amino acid sequences and the TMHMM Server v. 2.0 (Center for Biological Sequence Analysis, Technical University of Denmark, 2009, http://www.cbs.dtu.dk/services/ TMHMM/) was used to identify transmembrane helices. With the TatP 1.0 Server twin-arginine translocation (Tat) motifs in the $\mathrm{N}$-terminus were identified to predict protein localization in the cell (Bendtsen et al., 2005). The incorporation of selenocystein (SeCys) was examined by RNA loop predictions with Mfold version 3.2 (Mathews et al., 1999; Zuker, 2003). The RNA loop predicted in the 50-100 bp region downstream of the UGA-codon was compared with the consensus loop described by Zhang and Gladyshev (2005). Complete amino acid sequences of putative selenocystein-containing formate dehydrogenases were aligned to their homologues with CLUSTALX 1.81 (Kryukov and Gladyshev, 2004). SeCys incorporation was confirmed when the amino acid sequence aligned with conventional cystein in homologous proteins.

\section{Topic 3. Hypotheses for energy conservation mechanisms in butyrate oxidizers and propionate oxidizers}

\section{Butyrate oxidation by S. wolfei}

The electron transport in butyrate oxidation by S. wolfei was studied recently in a classical biochemical approach
(Müller et al., 2009). The electron transfer from butyrylCoA to external electron acceptors was found to be inhibited by trifluoperazine, a compound known to inhibit electron transfer to menaquinone by the respiratory complex I in Mycobacterium tuberculosis (Yano et al., 2006). Trifluoperazine also inhibited electron transfer from $\mathrm{NADH}$ to quinone analogues. An NADH-oxidizing enzyme complex was partially purified from the membrane fraction of $S$. wolfei. This activity was also found in the cytoplasmic fraction, especially after repeated treatment in the French Press cell, indicating that it is only superficially associated with the membrane (Müller et al., 2009). The enzyme complex contained several proteins which were analysed by peptide mass fingerprinting and were compared via the known gene sequence with redox enzymes found in other bacteria, i.e. an enzyme system similar to the confurcating hydrogenase of T. maritima (Schut and Adams, 2009). Moreover, this hydrogenase homologue of $S$. wolfei appeared to be associated with homologues of an NADHdependent formate dehydrogenase of Eubacterium acidaminophilum (Graentzdoerffer et al., 2003), a bacterium that can also grow in syntrophic association with partner organisms (Zindel et al., 1988). This enzyme complex could either act as a confurcating hydrogenase/ formate dehydrogenase or as a proton-pumping NADH dehydrogenase (NDH) (Müller et al., 2009). So far, it was not possible to show if this enzyme is directly linked to a transmembrane proton channel (Müller et al., 2009). But even if the enzyme lacks such a channel protons could be transferred via a menaquinone cycle (Fig. 1) as described above for E. coli (Jormakka et al., 2002). Whether the hydrogenase found really acts in a bifurcating manner as observed in T. maritima has still to be verified. So far, there is no indication of a ferredoxin-coupled redox reaction in butyrate oxidation by this bacterium.

Our results indicate that the 'Buckel-Thauer' reaction, i.e. a bifurcation of electrons from NADH with crotonylCoA and oxidized ferredoxin by the Bcd/EtfAB complex of C. kluyveri (Herrmann et al., 2008; Li et al., 2008), is not involved in butyrate oxidation by this bacterium. Until now, the function of etf genes in syntrophic butyrate degraders remains unclear. Possibly, the $\mathrm{Bcd} / \mathrm{EtfAB}$ complex is expressed when $S$. wolfei grows by dismutation of crotonate. Whether other butyrate oxidizers, e.g. S. aciditrophicus or $S$. buswellii, employ the Bcd/EtfAB complex in butyrate oxidation remains an open question at this time.

Another interesting feature of the hydrogenase homologue of $S$. wolfei is its association with a formate dehydrogenase (Müller et al., 2009). This supports older speculations that electrons from $\mathrm{NADH}$ oxidation are released as formate rather than hydrogen. The bacterium might even be able to choose which carrier it prefers, depending on the environmental conditions (Graentzdoerffer et al., 2003), e.g. whether a partner is present which 


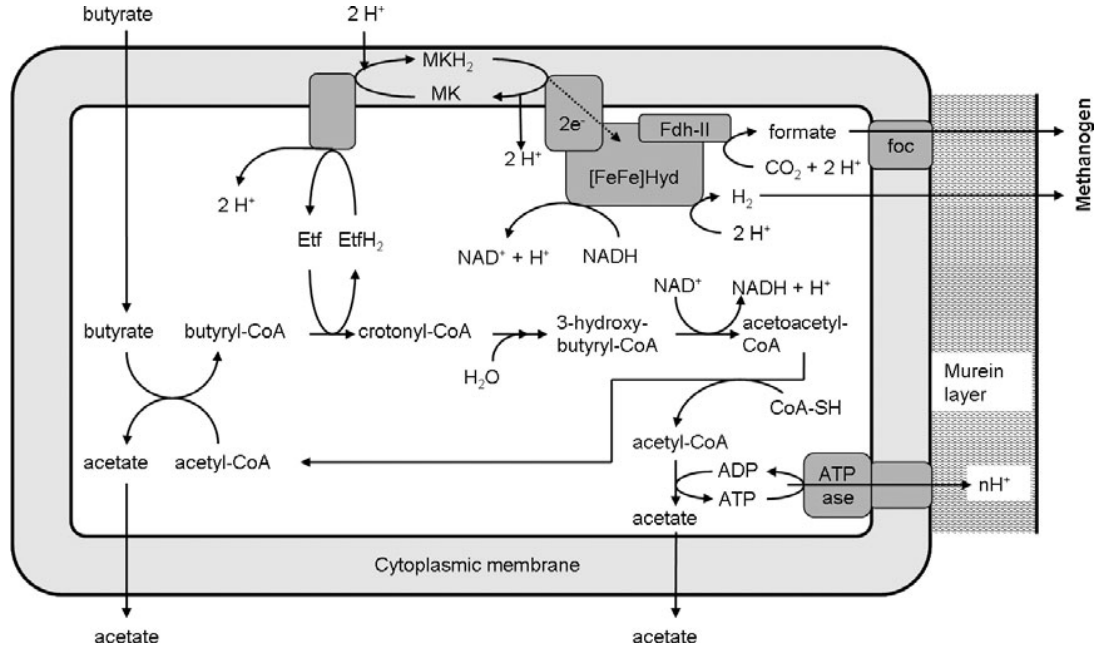

Fig. 1. Hypothetical energy-transforming mechanisms in the butyrate-degrading Syntrophomonas wolfei. The fatty acids acetate, butyrate and formate represent acetate ${ }^{-}+\mathrm{H}^{+}$, butyrate ${ }^{-}+\mathrm{H}^{+}$and formate ${ }^{-}+\mathrm{H}^{+}$ respectively. (foc) represents a formate transporter. consumes hydrogen, formate or both, and this preference might even differ between different butyrate oxidizers: co-culture experiments with $S$. bryantii and different partners (Dong et al., 1994) showed highest growth and substrate conversion rates with Methanospirillum hungatei which uses both hydrogen and formate, whereas co-cultures with the mainly formate-oxidizing Methanobacterium formicicum were slower, and there was no growth at all with the only hydrogen-consuming Methanobrevibacter arboriphilus. In contrast, it was shown earlier that $S$. wolfei grows in the presence of $M$. arboriphilus, although to a lower extent, indicating that $S$. wolfei can grow by interspecies hydrogen transfer only and formate plays only a minor role in electron transfer (Mcinerney et al., 1979; 1981).

Therefore, the electron transport during butyrate oxidation by $S$. bryantii might be different from that described above for $S$. wolfei, although the molecular prerequisites might be similar due to the close relatedness of both organisms. The formate dehydrogenase of $S$. bryantii was found to be membrane-bound and was most likely oriented to the periplasmic space whereas the partly membranebound hydrogenase was found in the cytoplasm and showed also activity with $\mathrm{NAD}^{+}$as electron acceptor (Dong and Stams, 1995). It was assumed that hydrogen is produced inside the cell while formate is produced outside. Additionally, an NADH dehydrogenase reacting with the tetrazolium dye MTT was measured, comparable to the described $\mathrm{NADH}$ :quinone oxidoreductase in $S$. wolfei which also reacts with MTT (Müller et al., 2009; N. Müller unpubl. data). It is tempting to speculate at this point that the described NADH dehydrogenase of $S$. wolfei could also be a bifurcating hydrogenase that couples NADHdependent proton reduction with quinone reduction by another molecule of NADH. One electron pair would then be used to reduce protons to form hydrogen and the other one would be transferred to the external formate dehydro- genase. If hydrogenase and/or NADH dehydrogenase are coupled to the formate dehydrogenase via a quinonemediated redox loop, two additional protons would be transferred outside the cell. Overall, proton consumption in the cytoplasmic space and proton release at the outside would result in a net proton gradient which in turn could drive menaquinol oxidation with $\mathrm{NAD}^{+}$or ADP phosphorylation by proton influx. This would require that formate and hydrogen both have to be kept at low concentration to allow the thermodynamically unfavourable reactions of $\mathrm{CO}_{2}$ reduction with quinols and proton reduction with $\mathrm{NADH}$. Although such a membrane-bound and quinone-oxidizing formate dehydrogenase has not yet been measured in $S$. wolfei, there are indications for such a system in its genome (Swol_0797-Swol_0799) (Table 2).

Although the formate/ $\mathrm{CO}_{2}$ couple and the hydrogen/ proton couple are both at the same redox potential under physiological conditions, the question remains whether they are really equivalent inside the cell as assumed earlier (Schink, 1997). Co-cultures of Moorella sp. strain AMP and Desulfovibrio sp. strain G11 with formate as substrate in co-culture with hydrogen-only consuming methanogens converted formate to methane (Dolfing et al., 2008). It was assumed that formate is oxidized outside the cytoplasmic membrane, $\mathrm{CO}_{2}$ and protons are released and electrons are shuttled to a membranebound hydrogenase facing the cytoplasm where protons are consumed (Dolfing et al., 2008). Thus, a net positive membrane potential could be formed without direct proton translocation but there is so far no proof that this reaction is coupled to energy conservation.

\section{Propionate oxidation by S. fumaroxidans and P. thermopropionicum}

The most difficult step in syntrophic propionate oxidation is the oxidation of succinate to fumarate. In the past, 


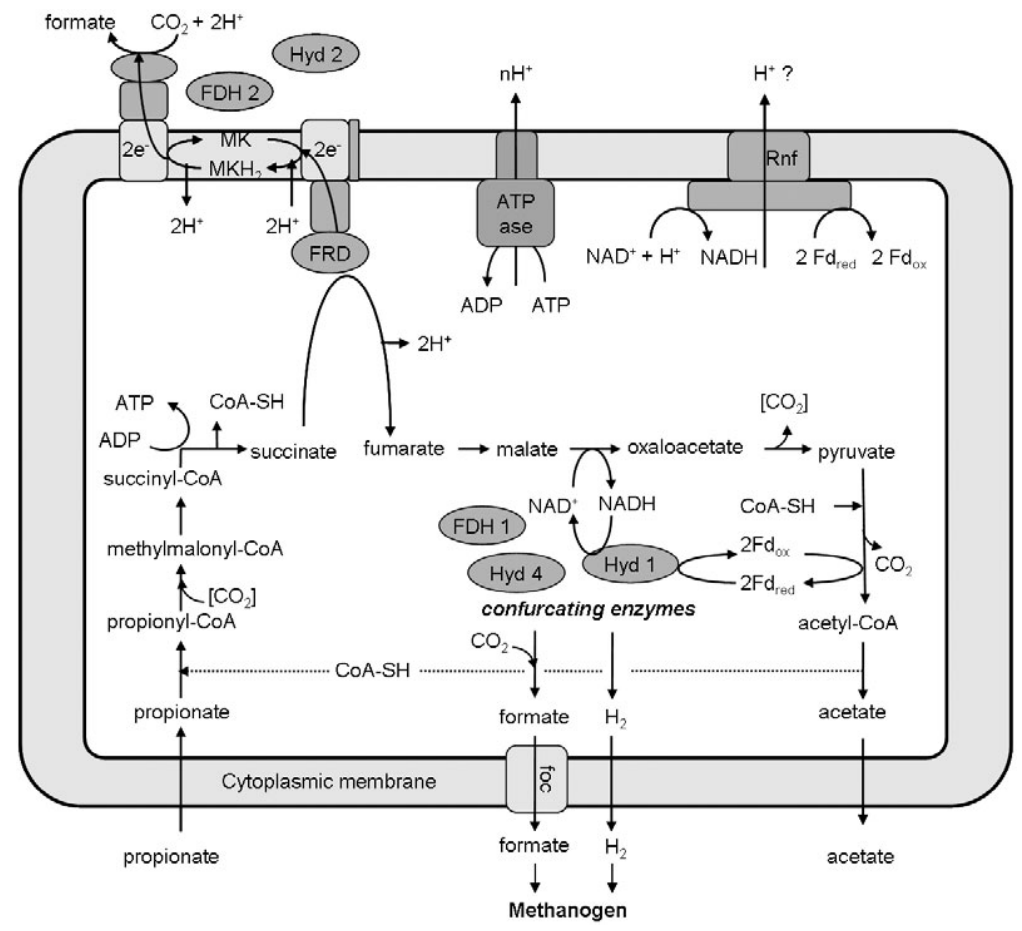

Fig. 2. Hypothetical energy-transforming mechanisms in the propionate-degrading Syntrophobacter fumaroxidans. The fatty acids acetate, propionate and formate represent acetate ${ }^{-}+\mathrm{H}^{+}$, propionate ${ }^{-}+\mathrm{H}^{+}$and formate ${ }^{-}+\mathrm{H}^{+}$respectively. (foc) represents a formate transporter, $\left[\mathrm{CO}_{2}\right]$ a biotin-bound carboxylic group. succinate dehydrogenases and fumarate reductases have been found to be similar based on their amino acid sequence (Lancaster, 2002). These authors classified fumarate reductases in five groups based on molecular composition. The fumarate reductase of $W$. succinogenes was classified within the group containing one hydrophobic subunit and two haem groups (Kröger et al., 2002). Our present gene analyses indicate that not only hydrophobic subunits of the fumarate reductase but also those of formate dehydrogenases of $W$. succinogenes (formate dehydrogenase delta subunits: WS0027, WS0736 and WS1148) contain haem groups and are homologous to cytochrome $b$.

Syntrophobacter fumaroxidans genome analysis revealed the presence of periplasmic formate dehydrogenases and hydrogenases (Fig. S1) as well as cytoplasmic fumarate reductases (Sfum_4092-4095, Sfum_19982000) which lack haem groups and a cytochrome $b$-like membrane-integrated domain. As such, fumarate reductases of $S$. fumaroxidans could not be classified within the five types described by Lancaster (2002). Instead, scattered over the genome, three cytochrome $b$ (cytb561; Sfum_0091, cytb5; Sfum_3227 and cytb; Sfum_2932) and three cytochrome $c$ homologous genes (Sfum_0090, Sfum_4047 and Sfum_1148) were found. Moreover, three genes with homology to cytochrome $b$ :quinone oxidoreductases were found (Sfum_0339, Sfum_3009 and Sfum_3051). Cytochrome $b$ and cytochrome $b$ :quinone oxidoreductases possibly function in a similar way as the cytochrome-containing membrane-integrated domains of fumarate reductases, hydrogenases and formate dehydrogenases of $W$. succinogenes (Fig. 2). Candidates for periplasmic formate or hydrogen oxidation are formate dehydrogenase 2, 3 and 4 and hydrogenase 2 (Fig. 2, Fig. S1). These proteins may bind periplasmic cytochrome $c$ and hydrophobic cytochrome $b$ for succinate oxidation or fumarate reduction. Probably they also interconvert hydrogen and formate via a cytochrome $c$ network, as proposed previously for the sulfate-reducing Desulfovibrio vulgaris Hildenborough (Heidelberg et al., 2004). Interconversion of hydrogen plus $\mathrm{CO}_{2}$ and formate by $S$. fumaroxidans was observed by Dong and Stams (1995) and de Bok and colleagues (2002).

Formate dehydrogenase 1 of $S$. fumaroxidans was previously characterized (de Bok et al., 2003). It oxidizes formate with benzyl viologen as artificial electron acceptor, but $\mathrm{NAD}^{+}$did not support oxidation of formate. Based on the amino acid sequence, this selenocysteincontaining formate dehydrogenase is similar to FDHII of $S$. wolfei and to the NADH-dependent formate dehydrogenase of Eubacterium acidaminophilum (Graentzdoerffer et al., 2003). Whether formate dehydrogenase 1 can couple oxidation of both $\mathrm{NADH}$ and ferredoxin to $\mathrm{CO}_{2}$ reduction in a manner analogous to proton reduction by the confurcating [FeFe]-hydrogenase of $T$. maritima was never tested. Based on our gene analysis, we hypothesize that hydrogenase 1 , hydrogenase 4 and formate dehydrogenase 1 can couple the oxidation of NADH generated from malate oxidation with the oxidation of reduced ferredoxin generated from pyruvate oxidation to produce 
hydrogen or formate (Table 2, Fig. 2). Especially transcription of genes coding for hydrogenase 1 (an [FeFe]hydrogenase) appears to be upregulated when metabolic conversions generate NADH and reduced ferredoxin (P. Worm, A.J.M. Stams, X. Cheng and C.M. Plugge, unpublished). In S. fumaroxidans the Rnf complex might be used to conserve the energy of ferredoxin oxidation with $\mathrm{NADH}$ reduction by exporting protons, thus equilibrating the ratio of reduced ferredoxin to NADH to 1:1.

Compared with $S$. fumaroxidans, the genome of $P$. thermopropionicum contains less formate dehydrogenaseand hydrogenase-coding genes (Table 2). However, for each metabolic task, several candidates are present just as in S. fumaroxidans. In order to reoxidize the NADH and reduced ferredoxin that are generated during propionate degradation, $P$. thermopropionicum likely uses the confurcating formate dehydrogenase (PTH_2645-2649) and the two confurcating [FeFe]-hydrogenases (PTH_13771379 and PTH_2010-2012) (Kosaka et al., 2008). The produced formate would be transported through the membrane via a formate transporter (PTH_2651) of which the gene is located in the operon coding for the cytoplasmic formate dehydrogenase. The produced hydrogen diffuses through the membrane and is used by the methanogen.

Also similar to $S$. fumaroxidans is the mechanism of reversed electron transfer via fumarate reductase, a menaquinone loop and a periplasmic formate dehydrogenase (PTH_1711-1714) or hydrogenase (PTH_17011704) (Kosaka et al., 2008). Cytoplasmic and periplasmic formate dehydrogenases and hydrogenases could be used to interconvert formate and hydrogen. Pelotomaculum thermopropionicum can grow only with a hydrogenusing methanogen as its syntrophic partner (Ishii et al., 2005); however, formate is likely to generate hydrogen.

The high number of formate dehydrogenase- and hydrogenase-encoding genes in $S$. fumaroxidans likely provides $S$. fumaroxidans with more back-up possibilities when formate and hydrogen concentrations vary according to the activity of the partner methanogen. In contrast to $S$. fumaroxidans, $P$. thermopropionicum lacks an Rnf cluster and ferredoxin-reducing hydrogenases and formate dehydrogenases. Syntrophobacter furmaroxidans might use these mechanisms as alternatives to reoxidize NADH and ferredoxin, possibly with the use of an electron potential via the Rnf cluster, when environmental conditions change.

\section{Concluding remarks}

Recent biochemical studies and genome analyses indicated that $S$. wolfei uses electron-transferring flavoproteins coupled to a menaquinone loop to drive endergonic butyryl-CoA oxidation, and $S$. fumaroxidans uses a periplasmic formate dehydrogenase, cytochrome $b$ :quinone oxidoreductases, a menaquione loop and a cytoplasmic fumarate reductase to drive endergonic succinate oxidation. Furthermore, we propose that confurcating [FeFe]hydrogenases in $S$. wolfei and $S$. fumaroxidans are involved in NADH oxidation to form hydrogen. For both $S$. wolfei and S. fumaroxidans, a similar function is proposed for a formate dehydrogenase which would result in simultaneous hydrogen and formate transfer from the fermenting bacterium to the hydrogen- and formate-consuming syntrophic partner. Syntrophobacter fumaroxidans and S. wolfei are proposed to produce hydrogen and formate in the cytoplasm. Pelotomaculum thermopropionicum and S. wolfei are proposed to contain a mechanism to convert hydrogen into formate which would allow growth with hydrogen-only using methanogens. These proposed energy-converting mechanisms need biochemical verification. We hypothesize that they are key in syntrophic propionate- and butyrate-degrading communities, as well as in other syntrophic communities.

\section{Acknowledgements}

The authors were financially supported by the German Research Foundation (DFG) and by the Earth and Life Sciences division (ALW) and Chemical Science division (CW) of the Netherlands Organization for Scientific Research (NWO).

\section{References}

Backiel, J., Juárez, O., Zagorevski, D.V., Wang, Z., Nilges, M.J., and Barquera, B. (2008) Covalent binding of flavins to RnfG and RnfD in the Rnf complex form Vibrio cholera. Biochemistry-US 47: 11273-11284.

Bendtsen, J.D., Nielsen, H., Widdick, D., Palmer, T., and Brunak, S. (2005) Prediction of twin-arginine signal peptides. BMC Bioinformatics 6: 167.

de Bok, F.A.M., Stams, A.J.M., Dijkema, C., and Boone, D.R. (2001) Pathway of propionate oxidation by a syntrophic culture of Smithella propionica and Methanospirillum hungatei. Appl Environ Microbiol 67: 1800-1804.

de Bok, F.A.M., Luijten, M.L.G.C., and Stams, A.J.M. (2002) Biochemical evidence for formate transfer in syntrophic propionate-oxidizing cocultures of Syntrophobacter fumaroxidans and Methanospirillum hungatei. Appl Environ Microbiol 68: 4247-4252.

de Bok, F.A.M., Hagedoorn, P.L., Silva, P.J., Hagen, W.R., Schiltz, E., Fritsche, K., and Stams, A.J.M. (2003) Two W-containing formate dehydrogenases $\left(\mathrm{CO}_{2}\right.$-reductases $)$ involved in syntrophic propionate oxidation by Syntrophobacter fumaroxidans. Eur J Biochem 270: 24762485.

Chabrière, E., Charon, M.H., Volbeda, A., Pieulle, L., Hatchikian, E.C., and Fontecilla-Camps, J.C. (1999) Crystal structures of the key anaerobic enzyme pyruvate: ferredoxin oxidoreductase, free and in complex with pyruvate. Nat Struct Biol 6: 182-190.

Dolfing, J., Jiang, B., Henstra, A.M., Stams, A.J.M., and Plugge, C.M. (2008) Syntrophic growth on formate: a new 
microbial niche in anoxic environments. Appl Environ Microbiol 74: 6126-6131.

Dong, X.Z., and Stams, A.J.M. (1995) Evidence for $\mathrm{H}_{2}$ and formate formation during syntrophic butyrate and propionate degradation. Anaerobe 1: 35-39.

Dong, X.Z., Cheng, G., and Stams, A.J.M. (1994) Butyrate oxidation by Syntrophospora bryantii in coculture with different methanogens and in pure culture with pentenoate as electron-acceptor. Appl Microbiol Biotechnol 42: 647-652.

Graentzdoerffer, A., Rauh, D., Pich, A., and Andreesen, J.R. (2003) Molecular and biochemical characterization of two tungsten- and selenium-containing formate dehydrogenases from Eubacterium acidaminophilum that are associated with components of an iron-only hydrogenase. Arch Microbiol 179: 116-130.

Gustafson, W.G., Feinberg, B.A., and Mcfarland, J.T. (1986) Energetics of beta-oxidation - reduction potentials of general fatty acyl-CoA dehydrogenase, electron-transfer flavoprotein, and fatty acyl-CoA substrates. J Biol Chem 261: 7733-7741.

Heidelberg, J.F., Seshadri, R., Haveman, S.A., Hemme, C.L., Paulsen, I.T., Kolonay, J.F., et al. (2004) The genome sequence of the anaerobic, sulfate-reducing bacterium Desulfovibrio vulgaris Hildenborough. Nat Biotechnol 22: 554-559.

Herrmann, G., Jayamani, E., Mai, G., and Buckel, W. (2008) Energy conservation via electron-transferring flavoprotein in anaerobic bacteria. J Bacteriol 190: 784-791.

Hoehler, T.M., Alperin, M.J., Albert, D.B., and Martens, C.S. (2001) Apparent minimum free energy requirements for methanogenic archaea and sulfate-reducing bacteria in an anoxic marine sediment. FEMS Microbiol Ecol 38: 33-41.

Ishii, S., Kosaka, T., Hori, K., Hotta, Y., and Watanabe, K. (2005) Coaggregation facilitates interspecies hydrogen transfer between Pelotomaculum thermopropionicum and Methanothermobacter thermoautotrophicus. Appl Environ Microbiol 71: 7838-7845.

Jormakka, M., Törnroth, S., Byrne, B., and Iwata, S. (2002) Molecular basis of proton motive force generation: structure of formate dehydrogenase-N. Science 295: 18631868.

Kosaka, T., Kato, S., Shimoyama, T., Ishii, S., Abe, T., and Watanabe, K. (2008) The genome of Pelotomaculum thermopropionicum reveals niche-associated evolution in anaerobic microbiota. Genome Res 18: 442-448.

Kröger, A., Biel, S., Simon, J., Gross, R., Unden, G., and Lancaster, C.R.D. (2002) Fumarate respiration of Wolinella succinogenes: enzymology, energetics and coupling mechanism. Biochim Biophys Acta Bioenerg 1553: 23-38.

Kryukov, G.V., and Gladyshev, V.N. (2004) The prokaryotic selenoproteome. EMBO Rep 5: 538-543.

van Kuijk, B.L.M., and Stams, A.J.M. (1996) Purification and characterization of malate dehydrogenase from the syntrophic propionate-oxidizing bacterium strain MPOB. FEMS Microbiol Lett 144: 141-144.

van Kuijk, B.L.M., Schlösser, E., and Stams, A.J.M. (1998) Investigation of the fumarate metabolism of the syntrophic propionate-oxidizing bacterium strain MPOB. Arch Microbiol 169: 346-352.

Kumagai, H., Fujiwara, T., Matsubara, H., and Saeki, K. (1997) Membrane localization, topology, and mutual sta- bilization of the rnfABC gene products in Rhodobacter capsulatus and implications for a new family of energycoupling NADH oxidoreductases. Biochemistry-US 36 : 5509-5521.

Lancaster, C.R.D. (2002) Succinate: quinone oxidoreductases: an overview. Biotech Biophys Acta Bioenerg 1553: 1-6.

Li, F., Hinderberger, J., Seedorf, H., Zhang, J., Buckel, W., and Thauer, R.K. (2008) Coupled ferredoxin and crotonyl coenzyme a $(\mathrm{COA})$ reduction with $\mathrm{NADH}$ catalyzed by the butyryl-CoA dehydrogenase/Etf complex from Clostridium kluyveri. J Bacteriol 190: 843-850.

Mclnerney, M.J., Bryant, M.P., and Pfennig, N. (1979) Anaerobic bacterium that degrades fatty acids in syntrophic association with methanogens. Arch Microbiol 122: 129-135.

Mclnerney, M.J., Bryant, M.P., Hespell, R.B., and Costerton, J.W. (1981) Syntrophomonas wolfei gen. nov. sp. nov, an anaerobic, syntrophic fatty acid-oxidizing bacterium. Appl Environ Microbiol 41: 1029-1039.

Mclnerney, M.J., Rohlin, L., Mouttaki, H., Kim, U., Krupp, R.S., Rios-Hernandez, L., et al. (2007) The genome of Syntrophus aciditrophicus: life at the thermodynamic limit of microbial growth. Proc Natl Acad Sci USA 104: 76007605.

Mclnerney, M.J., Struchtemeyer, C.G., Sieber, J., Mouttaki, H., Stams, A.J.M., Schink, B., et al. (2008) Physiology, ecology, phylogeny, and genomics of microorganisms capable of syntrophic metabolism. Ann N Y Acad Sci 1125: 58-72.

Mathews, D.H., Sabina, J., Zuker, M., and Turner, D.H. (1999) Expanded sequence dependence of thermodynamic parameters improves prediction of RNA secondary structure. J Mol Biol 288: 911-940.

Müller, V., Imkamp, F., Biegel, E., Schmidt, S., and Dilling, S. (2008) Discovery of a ferredoxin:NAD+-oxidoreductase (Rnf) in Acetobacterium woodii: a novel potential coupling site in acetogens. Ann NY Acad Sci 1125: 137-146.

Müller, N., Schleheck, C., and Schink, B. (2009) Involvement of NADH : acceptor oxidoreductase and butyryl-CoA dehydrogenase in reversed electron transport during syntrophic butyrate oxidation by Syntrophomonas wolfei. J Bacteriol 191: 6167-6177.

Nakanishi-Matsui, M., and Futai, M. (2008) Stochastic rotational catalysis of proton pumping F-ATPase. Philos Trans $R$ Soc Lond B Biol Sci 363: 2135-2142.

Nakayama, Y., Yasui, M., Sugahara, K., Hayashi, M., and Unemoto, T. (2000) Covalently bound flavin in the NqrB and NqrC subunits of $\mathrm{Na}^{+}$-translocating $\mathrm{NADH}$-quinone reductase from Vibrio alginolyticus. FEBS Lett 474: 165-168.

Richardson, D.J. (2000) Bacterial respiration: a flexible process for a changing environment. Microbiology 146: 551-571.

Schink, B. (1997) Energetics of syntrophic cooperation in methanogenic degradation. Microbiol Mol Biol Rev 61: 262-280.

Schink, B., and Stams, A.J.M. (2006) Syntrophism among prokaryotes. Prokaryotes 2: 309-335.

Schut, G.J., and Adams, M.W.W. (2009) The iron-hydrogenase of Thermotoga maritima utilizes ferredoxin and NADH synergistically: a new perspective on 
anaerobic hydrogen production. J Bacteriol 191: 44514457.

Stams, A.J.M., and Plugge, C.M. (2009) Electron transfer in syntrophic communities of anaerobic bacteria and archaea. Nat Rev Microbiol 7: 568-577.

Thauer, R.K., and Morris, J.G. (1984) Metabolism of chemotrophic anaerobes: old views and new aspects. Symp Soc Gen Microbiol 36 (Part 2): 123-168.

Thauer, R.K., Jungermann, K., and Decker, K. (1977) Energy-conservation in chemotropic anaerobic bacteria. Bacteriol Rev 41: 100-180.

Wallrabenstein, C., and Schink, B. (1994) Evidence of reversed electron-transport in syntrophic butyrate or benzoate oxidation by Syntrophomonas wolfei and Syntrophus buswellii. Arch Microbiol 162: 136-142.

Wofford, N.Q., Beaty, P.S., and Mclnerney, M.J. (1986) Preparation of cell-free-extracts and the enzymes involved in fatty-acid metabolism in Syntrophomonas wolfei. $J$ Bacteriol 167: 179-185.

Wu, C.G., Liu, X.L., and Dong, X.Z. (2006) Syntrophomonas cellicola $\mathrm{sp}$ nov., a spore-forming syntrophic bacterium isolated from a distilled-spirit-fermenting cellar, and assignment of Syntrophospora bryantii to Syntrophomonas bryantii comb. nov. Int J Syst Evol Microbiol 56: 2331-2335.

Yano, T., Li, L.S., Weinstein, E., Teh, J.S., and Rubin, H. (2006) Steady-state kinetics and inhibitory action of antitubercular phenothiazines on Mycobacterium tuberculosis type-II NADH-menaquinone oxidoreductase (NDH-2). $J$ Biol Chem 281: 11456-11463.

Zhang, Y., and Gladyshev, V.N. (2005) An algorithm for identification of bacterial selenocysteine insertion sequence elements and selenoprotein genes. Bioinformatics 21: 2580-2589.
Zhao, H.X., Yang, D.C., Woese, C.R., and Bryant, M.P. (1993) Assignment of fatty acid-beta-oxidizing syntrophic bacteria to Syntrophomonadaceae fam. nov. on the basis of $16 \mathrm{~S}$ rRNA sequence analyses. Int $J$ Syst Bacteriol 43: 630-630.

Zindel, U., Freudenberg, W., Rieth, M., Andreesen, J.R., Schnell, J., and Widdel, F. (1988) Eubacterium acidaminophilum sp. nov., a versatile amino acid-degrading anaerobe producing or utilizing $\mathrm{H}_{2}$ or formate - description and enzymatic studies. Arch Microbiol 150: 254-266.

Zuker, M. (2003) Mfold web server for nucleic acid folding and hybridization prediction. Nucleic Acids Res 31: 34063415.

\section{Supporting information}

Additional Supporting Information may be found in the online version of this article:

Fig. S1. Schematic representation of putative formate dehydrogenases, hydrogenases and Rnf complexes in butyrate-degrading Syntrophomonas wolfei (A) and Syntrophus aciditrophicus (B), and in propionate-degrading Syntrophobacter fumaroxidans (C) and Pelotomaculum thermopropionicum (D). Gene locus tag numbers and $\alpha-, \beta$ - and $\gamma$-subunits are indicated in small characters, predicted iron-sulfur clusters and metal binding sites are indicated in capitals. 'Formate' represent formate ${ }^{-}+\mathrm{H}^{+}$.

Please note: Wiley-Blackwell are not responsible for the content or functionality of any supporting materials supplied by the authors. Any queries (other than missing material) should be directed to the corresponding author for the article. 